\title{
The use of acetate sheets as records for chromatographic patterns
}

Taxonomists are often faced with marking and analysing chromatographic patterns of large samples of taxa. If sheets, $46 \times 57 \mathrm{~cm}$ are used, the recording of the position of compounds, their fluorescence and color under U.V. light and their color changes with clifferent chromogenic sprays is often cumbersome, if done on separate sheets.

The recording upon the original chromatogram is not advisable, since these can repeatedly be exposed to different vapor phases for color change, and can then be subjected to a chromogenic spray to find further color reactions. If, in such a case, the position of compounds on the original chromatogram has been marked, the previously drawn lines may suggest compounds that are not in fact visible.

My work of recording chromatographic patterns was greatly facilitated by superimposing a thin sheet of clear acetate (Grumbacher Tuffilm I96-I5o) upon the original chromatogram. With wax pencil or china marker the exact outline of the compounds can be recorded on the acetate and their coloration noted. This can also be done in a vapor chamber or under U.V. light. The original can again be subjected to $\mathrm{NH}_{3}$ or other vapors, U.V. light and finally be sprayed, without being cluttered with notations, and without shadow spots produced by finger marks. In two-dimensional chromatography the exact position and relation of the compounds to each other in the pattern to be recorded is greatly facilitated. Since the application point and front line are also noted on the acetate sheet, several of these can be superimposed and deviating patterns immediately recognized. The acetate is inexpensive, resistant to many vapors, easily stored and can be used as a permanent record.

Department of Botany, University of Michigan, Ann Arbor, Mich. (U.S.A.)

RAINER W. Scora

Received April 22nd, I963

J. Chromatog., I $3\left(\operatorname{Ig}_{4}\right) 25 \mathrm{I}$

\section{"Crossing" paper electrophoresis for the detection of complexes of enzymes with their inhibitors}

The inhibition of lysozyme activity occurs in the presence of high molecular weight acidic polymers. It was found that the following substances effectively inhibited lysozyme activity: heparin ${ }^{1-3}$, ribo-nucleic acid, deoxyribonucleic acid, hyaluronic acid, pneumococcus polysaccharide, Vi antigen, glutamyl polypeptide ${ }^{4}$. The inhibition was of the competitive type. The lysozyme-trypsine complex was isolated ${ }^{5}$ and the precipitation and aggregate formation of deoxyribonucleic acid with lysozyme was examined ${ }^{6}$.

A similar type of inhibition can be observed with ribonucleic acid, which is the inhibitor of deoxyribonuclease $\mathrm{f}^{7,8}$. The reaction between these inhibitors belonging to the group of macromolecular acid compounds and lysozyme or deoxyribonuclease has an electrostatic character. In our previous work, we have demonstrated with the help of "crossing" electrophoresis that inhibitors of macromolecular acidic polymer nature 